\title{
From Parallel SAT to Distributed SAT
}

\author{
Youssef Hamadi \\ Microsoft Research \\ 7 JJ Thomson Avenue \\ Cambridge United Kingdom \\ LIX École Polytechnique \\ F-91128 Palaiseau, France \\ youssefh@microsoft.com
}

\section{Summary}

This tutorial will present an overview of parallelism in SAT. It will start with a presentation of classical divide and conquer techniques, discuss their ancient origin and compare them to more recent portfolio-based algorithms. It will then present the impact of clause-sharing on their performances and discuss various strategies used to control the communication overhead. A particular technique used to control the classical diversification/intensification tradeoff will also be presented. Finally, perspectives will be given which will relate the current parallel SAT technologies to the expected evolution of computational platforms, leading to distributed SAT solving scenarios.

\section{References}

[1] Y. Hamadi, S. Jabbour, and L. Sais. Control-based clause sharing in parallel sat solving. In Proceedings of the 21st International Joint Conference on Artificial Intelligence (IJCAI 2009), pages 499-504, 2009.

[2] Y. Hamadi, S. Jabbour, and L. Sais. ManySAT: a parallel SAT solver. Journal on Satisfiability, Boolean Modeling and Computation, 6:245-262, 2009.

[3] Luis Gil, Paulo Flores, and Luis Miguel Silveira. PMSat: a parallel version of minisat. Journal on Satisfiability, Boolean Modeling and Computation, 6:71-98, 2008.

[4] G. Chu and P. J. Stuckey. Pminisat: a parallelization of minisat 2.0. Technical report, Sat-race 2008, solver description, 2008.

[5] M. Lewis, T. Schubert, and B. Becker. Multithreaded sat solving. In 12th Asia and South Pacific Design Automation Conference, 2007.

[6] G. Ringwelski, Y. Hamadi. Boosting distributed constraint satisfaction. In Proceedings of the 11th International Conference on Principles and Practice of Constraint Programming (CP 2005), Springer LNCS 3709, 549-562, 2005.

[7] Bernard Jurkowiak, Chu Min Li, and Gil Utard. A parallelization scheme based on work stealing for a class of sat solvers. Journal of Automated Reasoning, 34(1):73-101, 2005.

[8] Wahid Chrabakh and Rich Wolski. GrADSAT: A parallel sat solver for the grid. Technical report, UCSB Computer Science Technical Report Number 2003-05, 2003.

[9] W. Blochinger, C. Sinz, and W. Küchlin. Parallel propositional satisfiability checking with distributed dynamic learning. Parallel Computing, 29(7):969-994, 2003.

[10] Max Böhm and Ewald Speckenmeyer. A fast parallel sat-solver - efficient workload balancing. Annals of Mathematics and Artificial Intelligence, 17(3-4):381-400, 1996.

[11] H. Zhang, M. P. Bonacina, and J. Hsiang. Psato: a distributed propositional prover and its application to quasigroup problems. Journal of Symbolic Computation, 21:543-560, 1996. 